\title{
Role of Immune Complexes from Pacients with Different Clinical Forms of Schistosomiasis in the Modulation of In Vitro Granuloma Reaction
}

\begin{abstract}
Simone A Rezende, José R Lambertucci ${ }^{*}$, Alfredo M Goes/ ${ }^{+}$
Departamento de Bioquímica e Imunologia, Instituto de Ciências Biológicas *Departamento de Clínica Médica, Faculdade de Medicina, Universidade Federal de Minas Gerais, Caixa Postal 486, 30161-970 Belo Horizonte,

MG, Brasil

Schistosomiasis is a disease whose pathology is strongly related to the granulomatous reaction formed around parasite eggs trapped in host tissues. Studies have shown that the chronic intestinal form (INT) of this infection is associated with a variety of immunoregulatory mechanisms which lead to a diminished granulomatous reaction. Using an in vitro model of granuloma reaction, we show that immune complexes (IC) isolated from sera of INT patients are able to reduce granulomatous reaction developed by peripheral blood mononuclear cells (PBMC) from acute (AC), INT and hepatosplenic (HE) patients to soluble egg antigen (SEA)-conjugated polyacrylamide beads (PB-SEA). This inhibitory activity is also observed in cell proliferation assay of PBMC from INT and HE patients stimulated with SEA and adult worm antigen (SWAP). Furthermore, IC isolated from sera of patients with different clinical forms of the disease are also able to suppress INT patients PBMC reactivity. Therefore, our results show that circulating IC present in sera of patients with different clinical forms of schistosomiasis may downregulate PBMC reactivity to parasite antigens resulting in a diminished granuloma reaction to parasite eggs.
\end{abstract}

Key words: Schistosoma mansoni - granuloma - immune complexes - clinical forms

Schistosomiasis mansoni is a chronic disease caused by a parasitic helminth, Schistosoma mansoni. The disease-related pathology is characterized by a granulomatous hypersensitivity reaction against eggs trapped in the liver and intestines of the infected host (Boros 1989). The anti-egg inflammatory response has been shown to be highly regulated by suppressive mechanisms in long term chronic infections in which is observed a decline in soluble egg antigen (SEA)-induced response, spontaneous regression of the granuloma size, and a decrease of pathology (Colley 1981). This regulation has been associated to many factors such as cytokines, idiotype anti-idiotype interactions, supressor T cells, macrophages and immune com-

\footnotetext{
This investigation received financial assistance from Coordenação de Aperfeiçoamento de Pessoal de Nível Superior (CAPES), Conselho Nacional de Pesquisa (CNPq), Pró-Reitoria de Pesquisa da Universidade Federal de Minas Gerais (PRPp-UFMG) and Fundação de Amparo à Pesquisa de Minas Gerais (FAPEMIG). +Corresponding author. Fax: +55-31-441. 5963. E-mail: goes@mono.icb.ufmg.br

Received 16 April 1997

Accepted 30 June 1997
}

plexes (IC) (Montesano et al. 1989, Goes et al. 1991, 1994, Joseph \& Boros 1993, Rezende et al. 1993, Flores-Villanueva et al. 1994).

Using an in vitro model of granuloma reaction we have shown that IC isolated from sera of chronic intestinal form (INT-IC) patients are able to regulate the granulomatous hypersensitivity developed by chronic intestinal patients peripheral blood mononuclear cells (PBMC) to polyacrylamide beads conjugated to $S$. mansoni egg antigens (PB-SEA) (Goes et al. 1991). This suppressive effect was also observed in cell proliferation assay where INT-IC induced a significant suppression of PBMC proliferative response to parasite antigens from eggs and adult worm (SWAP) (Rezende et al. 1993).

In this study we were interested to know if ICinduced suppression was dependent on the clinical form from which IC and/or PBMC were obtained. To accomplish this question we determined the ability of INT-IC to affect the reactivity of PBMC obtained from patients with different clinical forms of schistosomiasis, i.e., acute, chronic intestinal and hepatosplenic patients. Furthermore, we studied the effect of IC obtained from patients with different clinical forms of schistosomiasis in the reactivity of PBMC obtained from INT patients. 


\section{MATERIALS AND METHODS}

Study population - Schistosomiasis patients were selected in Ambulatório Orestes Diniz and Fundação Nacional da Saúde based on clinical and parasitological stool examination for the presence of $S$. mansoni egg antigens by the quantitative Kato-Katz procedure (Katz et al. 1972).

Isolation of IC - A pool of schistosomiasis serum from patients with different clinical forms $(\mathrm{N}=10)$ was inactivated at $56^{\circ} \mathrm{C}$ for $30 \mathrm{~min}$ and IC were precipitated using polyethylene glicol 6000 (PEG, Sigma P-2139) at a final concentration of 4\% (Goes et al. 1991). PEG-induced precipitates were ressuspended in PBS to the original sera volume and the quantity of protein was determined by Bradford Method (Bradford 1976). In the following assays, PBMC were treated with $125 \mathrm{mg} / \mathrm{ml}$ of IC.

In vitro granuloma reaction - PBMC were isolated from heparinized blood by Ficoll-diatrizoate density gradient centrifugation as previously described (Goes et al. 1991) and cultured in RPMI medium containing $10 \%$ heat inactivated $\mathrm{AB}+$ human serum (RPMI 10\% AB). Antigen-conjugated polyacrylamide beads, ressuspended in culture medium, were distributed at 200 beads/well in 96 well flat-bottomed tissue culture plates together with $3 \times 10^{5} \mathrm{PBMC}$. In vitro granuloma reaction was evaluated on day 5 of culture by quantitation of cellular reactivity around the beads using a phase contrast inverted tissue culture microscope, as previously described (Doughty et al. 1987, Hirsch \& Goes 1996). Three hundred separated determinations of cellular reactivity were made for each experimental group. The total score was then summed and the resultant mean expressed as granuloma index $(\mathrm{GI}) \pm$ standard deviation $(\mathrm{SD})$. Granulomatous reactivity to PB-SEA was compared to the nonspecific binding of PBMC to polyacrylamide beads not conjugated to antigen (PB).

Cell proliferation assay - $3 \times 10^{5} \mathrm{PBMC}$ from schistosomiasis patients were cultured in 96 well flat-bottomed plates in RPMI 10\% AB. These cultures were done in medium alone (MED) and with $25 \mathrm{mg} / \mathrm{ml}$ of SEA or SWAP. As positive control cells were stimulated with $10 \mathrm{mg} / \mathrm{ml}$ of phytohemaglutinin A (PHA-P). For the last $18 \mathrm{hr}$ of incubation, cells were pulsed with $0.5 \mathrm{mCi} /$ well of tritiated thymidine (ICN Biomedicals Inc., Irvine, CA). On day 5 of culture, cells were harvested onto glass fiber paper and beta scintillation counting was determined. Data are expressed as the mean of cpm values of triplicate cultures \pm standard deviation (SD).

Statistics - Statistical significance was determined using Student's $t$-test and significance was determined with the use of $P$ values $<0.05$.

\section{RESULTS}

Effect of IC in the reactivity of PBMC obtained from patients with different clinical forms of schistosomiasis - Previous data have shown that IC obtained from INT patients are able to reduce the reactivity of PBMC from INT patients to parasite antigens (Goes et al. 1991, 1994, Rezende et al. 1993). In this work we were interested in studying if this suppression depends on the clinical form of the patients from which IC and/or PBMC were isolated. Toward this end, PBMC from patients with different clinical forms of schistosomiasis were obtained and we tested the ability of IC isolated from INT patients (INT-IC) to suppress in vitro granuloma reaction and cell proliferation to parasite antigens. On in vitro granuloma reaction, all patients presented a significant reaction to PB-SEA when compared to PB. As previously observed, INT-IC provoked marked inhibition of INT-PBMC granulomatous hypersensitivity to PB-SEA (35\%). Furthermore, significant suppression of in vitro granuloma reaction was observed when PBMC obtained from $\mathrm{AC}$ or HE patients were incubated in the presence of IC ( $25 \%$ and $31 \%$, respectively) (Fig. 1).

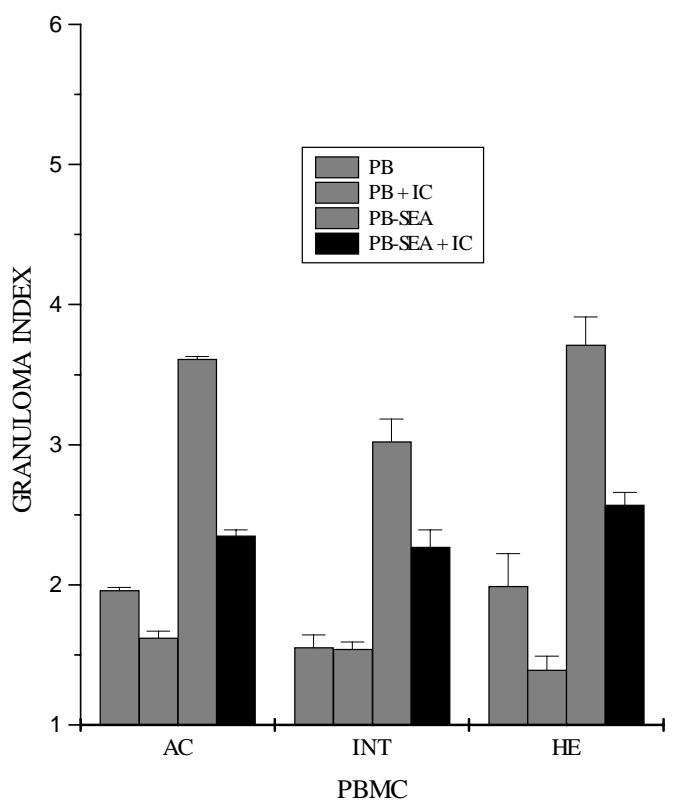

Fig. 1: $3 \times 10^{5}$ peripheral blood mononuclear cells (PBMC) from acute (AC), chronic intestinal (INT) or hepatosplenic (HE) patients were incubated in 96 well-flat-bottomed plates with no treatment or with $125 \mathrm{mg} / \mathrm{ml}$ of IC from INT patients (IC). These cells were co-cultured for 5 days with egg antigens conjugated to polyacrylamide beads (PB-SEA) or beads alone (PB). The granuloma index (GI) was evaluated by cellular reactivity around the beads. Data are reported as mean granuloma index \pm ED. N $=4$ (for each experimental group) 
Upon examination of the proliferative response of PBMC obtained from INT and HE patients to parasite antigens, we observed that IC isolated from INT patients provoked marked suppression of cell proliferation to both SEA and SWAP. The suppressive effect of IC was $42 \%$ on INT cells and $45 \%$ on HE cells when these cells were stimulated with SEA besides a suppression of $62 \%$ on INT cells and $60 \%$ on HE cells stimulated with SWAP (Fig. 2).

A different pattern of response was observed in acute patients PBMC as compared to that observed in INT and HE patients. In these patients INT-IC were not able to induce significant suppression on proliferative response of PBMC stimulated with SWAP or SEA (Fig. 2).

Effect of IC obtained from patients with different clinical forms of schistosomiasis in the reactivity of PBMC from chronic intestinal patients - In this assay we tested the effect of IC obtained from sera of patients with different clinical forms of schistosomiasis on in vitro granuloma reaction developed

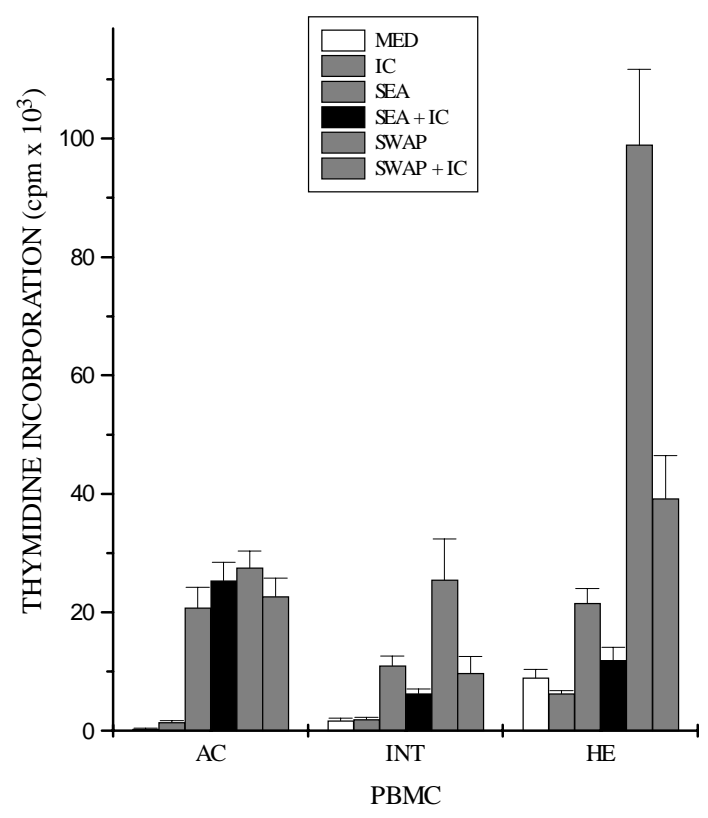

Fig. 2: $3 \times 10^{5}$ peripheral blood mononuclear cells (PBMC) from acute (AC), chronic intestinal (INT) or hepatosplenic patients (HE) were incubated in 96 well-flat-bottomed plates with no treatment or with $125 \mathrm{mg} / \mathrm{ml}$ of IC from INT patients (IC). These cells were co-cultured for 5 days with medium (MED), $25 \mathrm{mg} /$ $\mathrm{ml}$ of Schistosoma mansoni antigens from eggs (SEA) or adult worm (SWAP), $10 \mathrm{mg} / \mathrm{ml}$ of PHA (positive control) in cell proliferation assay. Then, PBMC were pulsed for the final $18 \mathrm{hr}$ with $0.5 \mathrm{mCi}$ of tritiated thymidine and incorporated radioactivity was determined. Data are reported as mean of cpm \pm ED. $\mathrm{N}=4$ (for each experimental group). $\mathrm{PHA}=12.345$. by PBMC from INT patients to PB-SEA. These IC were able to suppress in vitro granuloma reaction independent of the clinical form of the patient from which they were obtained. A suppression of $27 \%$ was observed in the granuloma reaction to PB-SEA when cells were incubated with IC obtained of AC patients and of $19 \%$ when cells were treated with IC obtained from HE patients (Fig. 3).

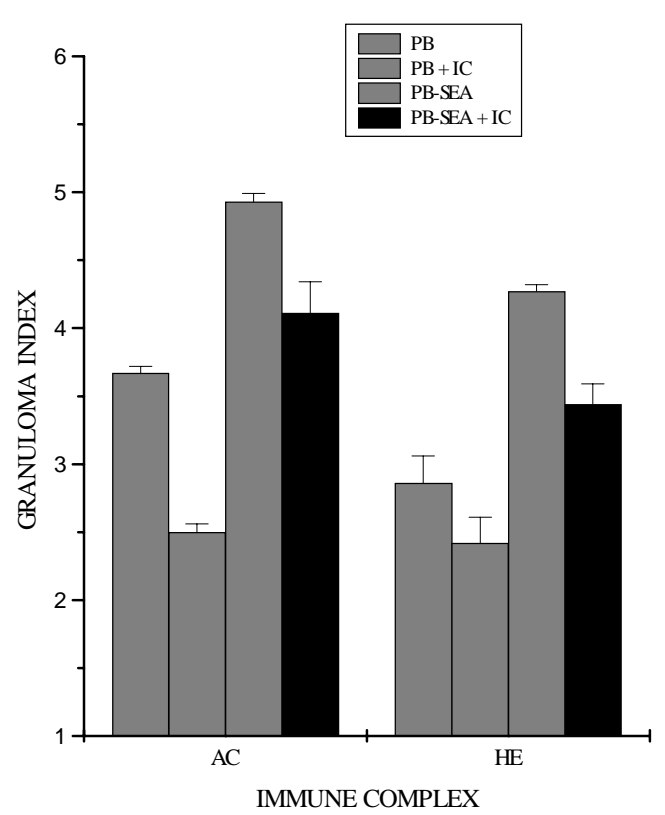

Fig. 3: $3 \times 10^{5}$ peripheral blood mononuclear cells (PBMC) from schistosomiasis patients were incubated in 96 well-flatbottomed plates with no treatment or with $125 \mathrm{mg} / \mathrm{ml}$ of IC obtained from acute (AC) or hepatosplenic (HE) patients. These cells were co-cultured for 5 days with egg antigens conjugated to polyacrylamide beads (PB-SEA) or beads alone (PB). The granuloma index (GI) was evaluated by cellular reactivity around the beads. Data are reported as mean granuloma index \pm ED for $\mathrm{N}=4$.

Furthermore we tested the effect of these IC in the proliferation of PBMC from INT patients and also detected a significant suppression in the proliferative response of PBMC to parasite antigens. The treatment of cells with IC obtained from AC patients caused a suppression of $19 \%$ in the response to SEA and of $43 \%$ to SWAP. Furthermore we observed that HE-IC induced a suppression of $31 \%$ and $59 \%$ in the proliferative response to SEA and SWAP, respectively (Fig. 4). 


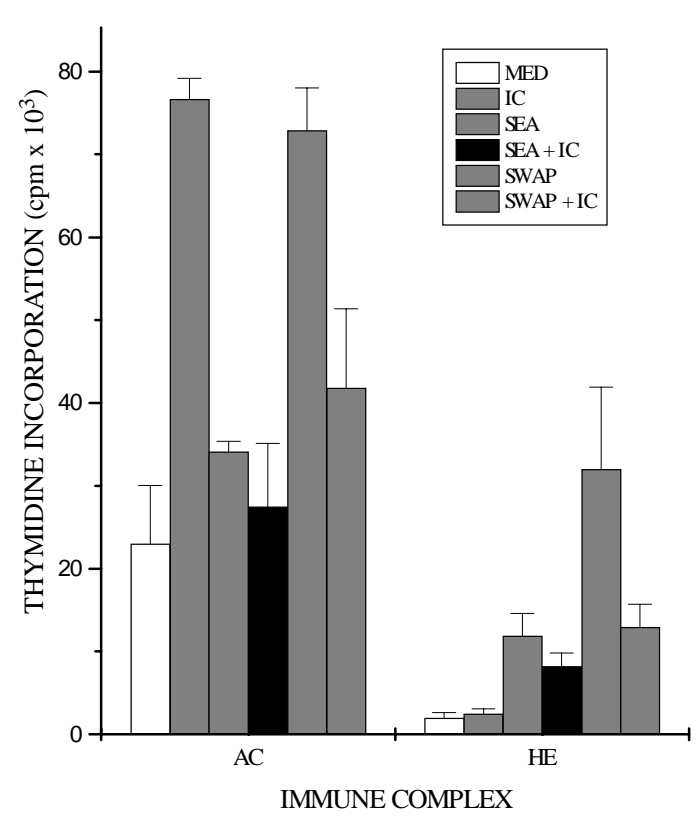

Fig. 4: $3 \times 10^{5}$ peripheral blood mononuclear cells (PBMC) from chronic intestinal patients (INT) were incubated in 96 well-flat-bottomed plates with no treatment or with $125 \mathrm{mg} / \mathrm{ml}$ of IC from acute (AC) or hepatosplenic (HE) patients. These cells were co-cultured for 5 days with medium (MED), $25 \mathrm{mg} /$ $\mathrm{ml}$ of Schistosoma mansoni antigens from eggs (SEA) or adult worm (SWAP), $10 \mathrm{mg} / \mathrm{ml}$ of PHA (positive control) in cell proliferation assay. Then, PBMC were pulsed for the final $18 \mathrm{hr}$ of culture with $0.5 \mathrm{mCi}$ of tritiated thymidine and incorporated radioactivity was determined. Data are reported as mean of cpm $\pm \mathrm{ED}$ for $\mathrm{N}=4$. $\mathrm{PHA}=15.300$.

\section{DISCUSSION}

Granulomatous reaction around parasite eggs trapped in host tissues is a prominent pathologic lesion in schistosomiasis. In the chronic phase, regulation of this lesion occurs, resulting in smaller anti-egg granulomas. Recent studies have demonstrated that several immunoregulatory mechanisms may be operative in human schistosomiasis. The possible interference of IC with cellular defense mechanisms against $S$. mansoni has been suggested by others (Butterworth et al. 1977, Kamal \& Higashi 1982). Afterwards, studies on in vitro granuloma reaction have shown that IC obtained from sera of INT patients are able to suppress INT patients PBMC reactivity to PB-SEA and PBSWAP (Goes et al. 1991, 1994, Rezende et al. 1993). This suppressive effect was also observed in the proliferative response of PBMC to parasite antigens (Rezende et al. 1993).

In this work we studied if IC-induced suppression was related to the clinical form of the schistosomiasis patients from which IC and/or PBMC were isolated. Our results demonstrated that IC obtained from sera of INT patients (INT-IC) were able to suppress in vitro granuloma reaction developed by PBMC from AC, INT and HE patients to $S$. mansoni egg antigens conjugated to polyacrylamide beads. A decrease in cell proliferation to SEA and SWAP was also observed when PBMC from INT and HE patients were incubated with INT-IC. Studies on patients with different clinical forms of schistosomiasis have shown that most acute patients are high responders to SEA and moderate responders to SWAP. Meanwhile, HE and INT patients are, in general, less responsive to both antigens (Colley et al. 1986). Therefore, the inability of IC to suppress cell proliferation to SEA and SWAP may be related to the ineffectiveness of immunoregulatory mechanisms to egg antigens in acute patients. Studies on the cytokine pattern of PBMC obtained from patients with different clinical forms of schistosomiasis upon IC stimulation are in progress in our laboratory and may be useful to the understanding of these processes (unpublished observations).

The mechanism by which IC down-modulates cell reactivity to $S$. mansoni antigens still awaits further elucidation. In this study, we demonstrated that IC obtained from patients with different clinical forms of schistosomiasis are able to suppress INT PBMC reactivity both on in vitro granuloma reaction and cell proliferation assay. Goes et al. (1991) have shown that the suppression of in vitro granuloma reaction is dependent on macrophages and IC composed of intact immunoglobulin (Ig). In that system, IC formed with $\mathrm{F}\left(\mathrm{ab}_{2}\right)$ IgG presented no regulatory activity, showing that the interaction of IC with macrophages depends on the Fc portion of the antibody molecule. We believe that IC-induced suppression of cell proliferative response may function in a different way, since these systems work differently. Studies on in vitro granuloma reaction have shown that this reaction is dependent on $\mathrm{CD}^{+}$cells and macrophages, and independent of B cells (Doughty et al. 1987). Otherwise, B cells are involved in processing and presentation of antigens in proliferative responses (Vidard et al. 1996) and may have their processing capability changed by the binding of Igs that form part of IC (Watts \& Lanzavecchia 1993). Therefore, we believe that, in cell proliferation assay, the binding of IC to Fc receptors on antigen-specific $\mathrm{B}$ cells might alter the presentation capability of these cells, causing a different pattern of response to different antigens. Then, the composition of IC may interfere in the response of antigenspecific B cells and be responsible for the antigenspecific effect observed in acute patients.

Recent data from our laboratory indicate that IC have the capacity to induce prostaglandin E pro- 
duction by macrophages and thus inhibit the initial events involved in granuloma formation (Goes et al. 1991, 1994). However, the characterization of immunoregulatory events and cytokines induced by IC is incomplete. Studies performed in our laboratory have demonstrated the involvement of cytokines in this system (unpublished observations). This suppression seems to be related to an increase in IL-10 production and a decrease in TNF-a production by mononuclear cells from schistosomiasis patients. This pattern of cytokines may influence the expression of adhesion molecules, cellular recruitment and lymphocyte activation, leading to the suppression of granulomatous reaction.

Taken together, our data suggest that IC obtained from patients with different clinical forms of schistosomiasis are able to modulate PBMC reactivity to $S$. mansoni eggs. Other suppressive mechanisms have been described in acute and HE patients but the fact that INT patients show a decreased granuloma reaction led us to suppose that these mechanisms, including IC-induced suppression, are more pronounced in these patients.

\section{REFERENCES}

Boros DL 1989. Immunopathology of Schistosoma mansoni infection. Clin Microbiol Rev 2: 250-280.

Bradford MM 1976. A rapid and sensitive method for the quantification of microgram quantities of protein utilizing the principle of protein-dye binding. Ann Biochem 72: 327-331.

Butterworth AE, Remold VH, David JR, Franks D, David PH, Sturrock RF 1977. Antibody-dependent eosinophil-mediated damage to $51_{\mathrm{Cr}}$-labeled schistosomula of Schistosoma mansoni: mediation by IgG, and inhibition by antigen-antibody complexes. $J$ Immunol 3: 2230-2236.

Colley DG 1981. Immune responses and immunoregulation in experimental and clinical schistosomiasis, p. 1-83. In JM Mansfild, Parasitic Diseases. Marcel Decker Inc., New York.

Colley DG, Garcia AA, Lambertucci JR, Parra JC, Katz N, Rocha RS, Gazzinelli G 1986. Immune responses during human schistosomiasis. XII. Differential responsiveness in patients with hepatosplenic disease. Am J Trop Med Hyg 35: 793-802.

Doughty BL, Goes AM, Parra JC, Rocha RS, Katz N, Colley DG, Gazzinelli G 1987. Granulomatous hypersensitivity to Schistosoma mansoni egg antigen in human schistosomiasis. I. Granuloma formation and modulation around polyacrylamide antigen-conjugated beads. Mem Inst Oswaldo Cruz 82: 47-54.

Flores-Villanueva PO, Harris TLS, Ricklan DE, Stadecker MJ 1994. Acessory cell signals regulate Th-cell responses: from basic immunology to a model of helminthic disease. J Immunol 152: 571574.

Goes AM, Gazzinelli G, Rocha R, Katz N, Doughty BL 1991. Granulomatous hypersensitivity to Schistosoma mansoni egg antigens in human schistosomiasis. III. In vitro granuloma modulation induced by immune complexes. Am J Trop Med Hyg 4: 434443.

Goes AM, Rezende SA, Gazzinelli G, Doughty BL 1994. Granulomatous hypersensitivity to Schistosoma mansoni egg antigens in human schistosomiasis IV. A role for prostaglandin induced inhibition of in vitro granuloma formation. Parasite Immunol 16: 11-18.

Hirsch C, Goes AM 1996. Characterization of fractionated Schistosoma mansoni soluble adult worm antigens that elicit human cell proliferation and granuloma formation in vitro. Parasitol 112: 529-535.

Joseph AL, Boros DL 1993. Tumor necrosis factor plays a role in Schistosoma mansoni egg-induced granulomatous inflammation. J Immunol 151: 5461-5471.

Kamal KA, Higashi GI 1982. Suppression of mitogeninduced lymphocyte transformation by plasma from patients with hepatosplenic schistosomiais mansoni: role of immune complexes. Parasite Immunol 4: 283298.

Katz N, Chaves A, Pellegrino J 1972. A simple device for quantitative stool thick-smear technique in schistosomiais mansoni. Rev Inst Med Trop São Paulo 14: 397-400.

Montesano MA, Lima MS, Correa-Oliveira R, Gazzinelli G, Colley DG 1989. Immune responses during human schistosomiasis mansoni. Idiotypic differences in antibody preparations from patients with different clinical forms of infection. J Immunol 142: 25012505.

Rezende SA, Miranda TCL, Ferreira MG, Goes AM 1993. In vitro granuloma modulation induced by immune complexes in human schistosomiasis mansoni. Braz J Med Biol Res 26: 207-211.

Vidard L, Kovacsovics-Bankowski M, Kraeft S, Chen LB, Benacerraf B, Rock KL 1996. Analysis of MHC class II presentation of particulate antigens by $\mathrm{B}$ lymphocytes. J Immunol 156: 2809-2818.

Watts C, Lanzavecchia A 1993. Suppressive effect of antibody on processing of T cell epitopes. J Exp Med 178: 1459-1463. 